\title{
Epidemiological Maxillofacial Injuries in Road Traffic Accidents: A Peripheral Hospital Based Study
}

Satadal Mondal, ${ }^{1}$ Indranil Sen, ${ }^{1}$ Rabi Hembrom, ${ }^{1}$ Swagato Roy, ${ }^{1}$ Rupam Sinha, ${ }^{1}$ Mayur Nair, ${ }^{1}$ Tapas Kumar Mahata ${ }^{1}$

\begin{abstract}
$\underline{\text { Introduction }}$
The aetiology of maxillofacial fractures is greatly influenced by geographic location, socioeconomic status of the cohort, and the period of investigation. The aim of this study is to analyze and identify characteristics of maxillo-facial fractures that took place in and around Midnapore- Kharagpore city of West Bengal and who presented to a peripheral medical college hospital during a period of 1 year.

Materials and Methods

A detailed database analysis was performed based on data collected from the patients of Road Traffic Accidents (RTA) with sustained facial trauma admitted to General Surgery and Otorhinolaryngology ward of a peripheral medical college hospital. Detailed clinical examination as well as radiological data was collected.

$\underline{\text { Results }}$

The highest frequency of maxillo-facial injury due to RTA was among the young adults 18-40 years. Most common type of injury encountered is abrasion (44\%) followed by bruise and closed fracture. Mandible is the most common bone to get fractured and most common type of Le fort type is Type II. Significant number of patients having RTA were young adults under the influence of alcohol riding in two-wheeler .
\end{abstract}

\section{$\underline{\text { ABSTRACT }}$}

\section{Conclusion}

With the increasing incidence of RTA awareness must be created concerning safety rules and more policies need to be addressed. $\underline{\text { Keywords }}$

Maxillofacial Injuries; Accidents, Traffic.

$\mathrm{M}$ axillofacial injuries constitute a huge disease burden to the present society. They are clinically as well as aesthetically important owing to their close vicinity to vital structures and cosmetic importance. Moreover, Maxillofacial (MF) fractures are often associated with severe morbidity, loss of function, disfigurement, and significant financial cost. ${ }^{1-3}$

The patterns of maxillofacial fracture presentation

1 - Department of Otorhinolaryngology, Midnapore Medical College Vidyasagar Road Midnapore

\section{Corresponding author:}

Dr Swagato Roy

email: royshin11@gmail.com are consistently influenced by geographic area, socioeconomic status of the cohort, and the period of investigation. According to reports of developing nations, traffic accidents are the main cause of maxillofacial fractures, while data from developed countries pointed to assaults being considered the most frequent etiology of such fractures. ${ }^{3-5}$

With regard to the anatomical sites, mandibular and zygomatic complex fractures account for the majority of all facial fractures and their occurrence varies according to the mechanism of injury and demographic factors, particularly, gender and age. The coordinated and sequential collection of information concerning demographic patterns of maxillofacial injuries may assist health care providers to record detailed and regular 
data of facial trauma. Consequently, an understanding of the cause, severity and temporal distribution of maxillofacial trauma permits clinical and research priorities to be established for effective treatment and prevention of those injuries.

Since face is the most exposed and unprotected part of the skeleton, the psychological impact of disfigurement can be devastating. The aim of this study is to describe the epidemiological profile of maxillo-facial fractures that occurred in and around Midnapore and who presented in the medical college hospital there during a period of one year (September 2017 to August 2018) .

\section{Materials and Method}

This was an ambispective study of the patients presenting with maxillo-facial fractures attending the General Surgery and Otorhinolaryngology ward of a peripheral medical college hospital over a period of 1 year. After initial management of the casualty ward, detailed histories were obtained either from the accident victim or from the accompanying persons or police personnel, in cases where the victims were not able to communicate due to medical reasons. The study included all cases of RTA attending the general emergencies with injuries of the MF region except, those who were on immediate life-risk or who did not wish to give the details and who were brought dead.

Each patient underwent a clinical examination as per proforma designed that was specifically developed to investigate the epidemiological features of MF trauma.

Table I: Types of injuries $(\mathbf{n}=\mathbf{2 0 0})$

\begin{tabular}{|l|c|c|}
\multicolumn{1}{|c|}{$\begin{array}{c}\text { TYPE OF } \\
\text { INJURY }\end{array}$} & $\begin{array}{c}\text { NO. OF } \\
\text { PATIENTS }\end{array}$ & PERCENTAGE \\
\hline Abrasion & 88 & $44 \%$ \\
\hline Bruise & 72 & $36 \%$ \\
\hline Fracture (closed) & 22 & $11 \%$ \\
\hline Fracture (open) & 16 & $8 \%$ \\
\hline $\begin{array}{l}\text { Injury requiring } \\
\text { Tracheostomy }\end{array}$ & 2 & $1 \%$ \\
\hline
\end{tabular}

\section{(Annexure)}

Age groups are divided into children $(<10$ years $)$, adolescent (10-17 years), young adults (18-40 years), adults (41-65 years), elderly ( $>65$ years). Type of injury was classified as Abrasion, Bruise, Closed fracture, Open fracture, Injury requiring Emergency Tracheostomy. Anatomical distribution of maxillofacial fractures was classified as Zygomatico-maxillary complex, Nasal bone, Naso-orbito-ethmoid, Mandible. Mandibular fractures included Body, Angle, Condyle, Symphysis, Ramus, Alveolar process, Coronoid Process.

\section{Results}

A total of 200 patients presenting with 237 maxillofacial fractures were analyzed. The ratio of male to female was 2.125:1. Patients' age ranged from 0 to 88 years (mean age $28 \pm 16$ yrs ). The most common age group affected was between 18 to 40 years i.e., the young adults, among them 56 were male and 32 were female. Next common age group was adults of 41- 65 years ( $29 \%$ of total RTA) . The least common age group were the elderly ( $>65$ years) comprised of only $5.5 \%$ of the total RTA. Distribution of types of injury is depicted in Table I.

Maximum number of RTA were seen in the two wheeler drivers $(62 \%)$ of whom alcohol smell was present in the breath of $79 \%$, followed by four wheelers (17\%) with alcohol smell present among $75 \%$ of the drivers. Least number of accidents was encountered with heavy vehicles $(6 \%)$ with alcohol smell present among $67 \%$ of the drivers.

In the study most common type of bone involved was mandible which was $68 \%$ of total RTA (Fig. 1). Second most common type was maxilla which was $16 \%$ of total RTA. Zygomatic bone involved $11 \%$ of the total fractures. Naso-Orbito-Ethmoid (NOE) fractures was seen only in $5 \%$ of total RTA. Common sites of mandibular fractures have been depicted in Table II. Fracture distribution in Middle third of the face is tabulated in Table III.

The zygomatic-maxillary complex (ZMC) fracture (Fig.2) was the most commonly encountered fracture among the middle third fractures $49.4 \%$, followed by 
Table II: Sites of mandibular fractures $(n=200)$

\begin{tabular}{|c|c|c|}
\hline FRACTURE SITE & PREVALENCE & FREQUENCY \\
\hline Body & $\mathbf{4 7 \%}$ & 94 \\
\hline Angle & $\mathbf{2 6 \%}$ & 52 \\
\hline Condyle & $19 \%$ & 38 \\
\hline Symphysis & $\mathbf{5 \%}$ & $\mathbf{1 0}$ \\
\hline Ramus & $\mathbf{2 \%}$ & 4 \\
\hline Alveolar & $\mathbf{1 \%}$ & 2 \\
\hline Coronoid Process & 0 & 0 \\
\hline
\end{tabular}

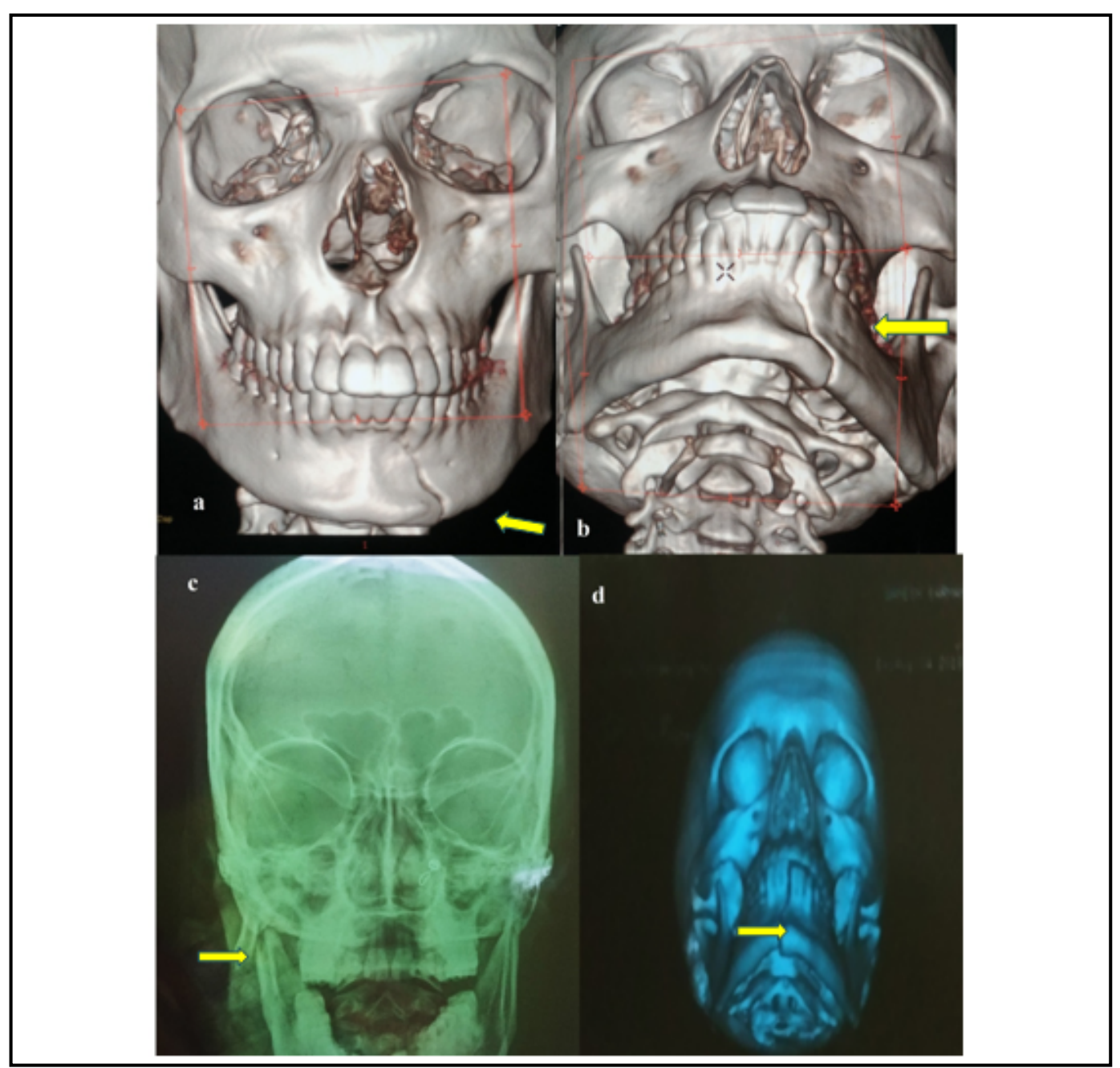

Fig. 1. a \& b : Fracture of the body of mandible; c: fracture at the condyle of mandible; $d$ : fracture at the symphysis of the mandible 
Table III: Distribution of fracture types in middle third of the face $(n=200)$

\begin{tabular}{|c|c|c|}
\hline FRACTURE TYPE & PERCENTAGE & FREQUENCY \\
\hline Le Fort I & $6 \%$ & 12 \\
\hline Le Fort II & $12 \%$ & 24 \\
\hline Le Fort III & $3.50 \%$ & 7 \\
\hline ZMC & $46 \%$ & 92 \\
\hline Dentoalveolar & $28 \%$ & 56 \\
\hline Palatal Split & $2 \%$ & 4 \\
\hline Nasal & $1.50 \%$ & 3 \\
\hline NOE & $1 \%$ & 2 \\
\hline
\end{tabular}

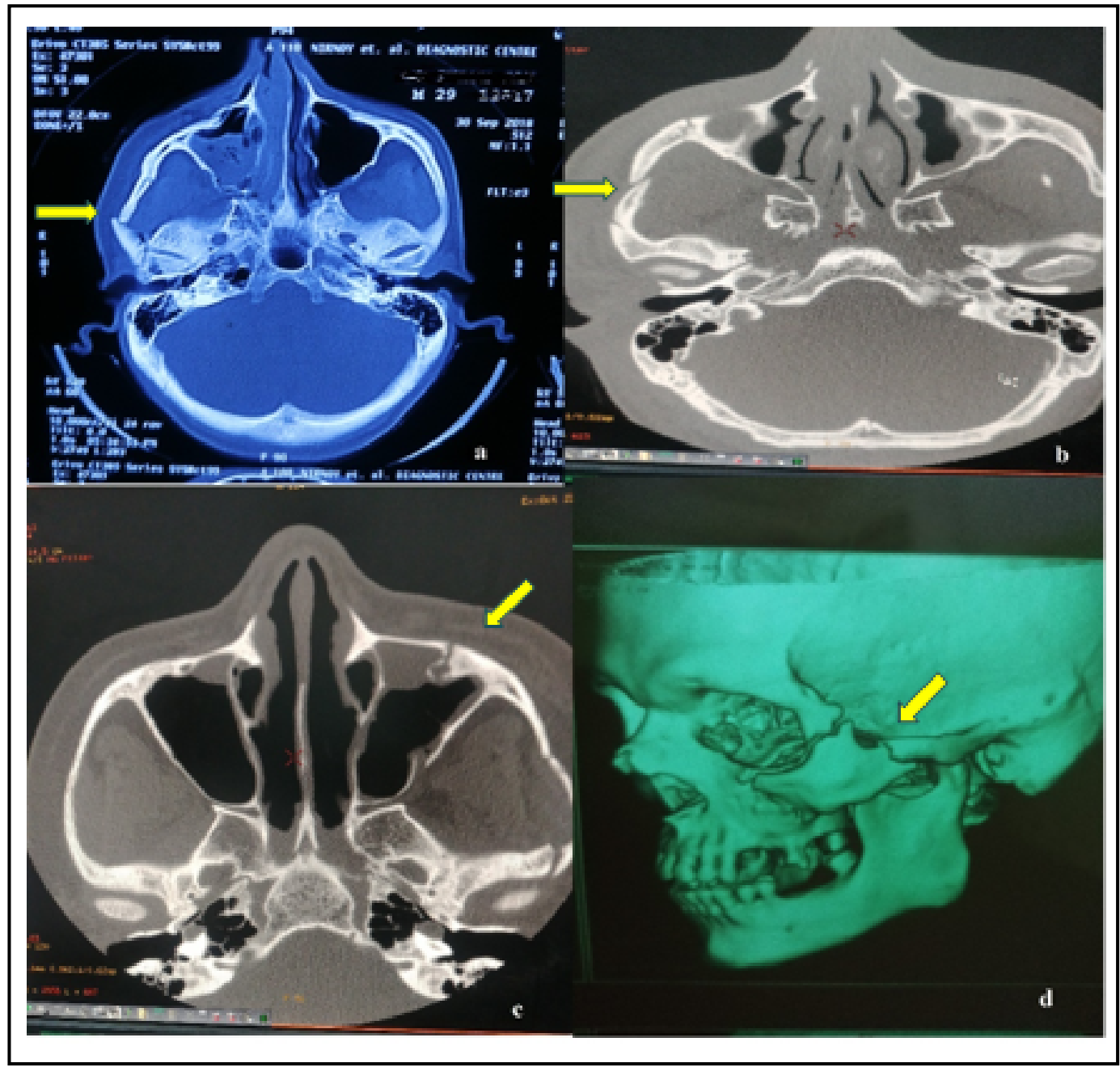

Fig. 2. a, b, c \& d : showing fracture of the zygomatic-maxillary complex fracture 
dento-alveolar fracture which was $28.3 \%$ of the middle third fractures. Among the Le Fort fractures, type II was the commonest which was $12.1 \%$ and only $0.6 \%$ of the fractures was seen in Naso-Orbito-Ethmoid (NOE) region.

\section{Discussion}

We studied a total of 200 patients who presented with maxillofacial fractures among whom there were $68 \%$ male and 32\% female (Male:female $=2.125: 1$ ). In earlier studies done by Gali et al 6, where there was a male predisposition (79.4\%) and that by Garkoti et al. ${ }^{7}$, where they found male incidence to be $80.77 \%$ and females $19.23 \%$.

Majority of the accidents were encountered among two-wheeler riders $(62 \%)$ of whom $79 \%$ were under alcohol intoxication which were proved by breath test.

The age group most commonly affected was that of $18-40$ years $(44 \%)$. In the study by Sawhney and Ahuja, ${ }^{8} 77 \%$ patients were in the age group of $16-45$ yrs .Garkoti et. $\mathrm{al}^{7}$ also got similar clustering of cases in the 20-30 age group. However in the study by Adeyemo et al. ${ }^{9}$ the most vulnerable age group was found $21-30$ years. In another study by Motamedi et $\mathrm{al}^{10}$, the most common age group affected was 20-29 year.

In our study, the most common site of fracture was the zygomaticomaxillary complex $(46 \%)$ followed by the Dento-alveolar (28\%). However, Dutta et $\mathrm{al}^{11}$ found that nasal bones were the most commonly fractured $(26.3 \%)$. Gali et al. ${ }^{6}$ found mandible to be commonly fractured (41.7\%), which was also supported by studies done by Sawhney et al. ${ }^{8}$ In the study of Adeyemo et. al ${ }^{9}$ most of the fractures of maxillofacial skeleton were of the mandible, the findings comparable to other reports. ${ }^{12,13-15}$ The mobility of the mandible and the fact that it has less bony support than the maxilla have been implicated. ${ }^{16,17}$ Dentoalveolar and condylar fractures were reported to be less in Nigerian patients. . $^{18,12,13-15,19}$

Dental/dento-alveolar injury is frequently overlooked in surveys that review maxillofacial injury. ${ }^{20-22}$ Only the analyses of a large number of injuries reveals the risk of suffering from dento-alveolar trauma. ${ }^{20-22}$ Gassner et $\mathrm{a}^{21}$ in a large series of 9,543 patients with 21,067 maxillofacial injuries reported an incidence of $49.9 \%$ of dentoalveolar injuries among their patients. Gassner et $\mathrm{al}^{22}$ in another large series of craniomaxillofacial trauma in 3,385 children younger than 15 years of age reported an incidence of $76.3 \%$ cases of dentoalveolar injuries. Midfacial bone fractures especially LeFort types and orbital floor fractures were reported to be commoner than mandibular fracture ${ }^{21,22}$ in contrast to Nigerian

\section{Conclusion}

The highest frequency of head injury was among the young adults $18-40$ years (44\%) Overall male to female ratio was 2.125:1. Significant number of patients having RTAs were young adults under the influence of alcohol riding two-wheeler. The most common structure to be injured in middle third of the face was zygomaticmaxillary complex fracture

Awareness must be created concerning safety rules and especially targeted at the high-risk groups which includes the most economically productive age group.

New policies need to be addressed for better road side assistance, road safety education and emergency treatment protocol for both para-medical and medical staff.

\section{References}

1. Down KE, Boot DA, Gorman DF. Maxillofacial and associated injuries in severely traumatized patients: implications of a regional survey. Int J Oral Maxillofac Surg. 1995; 24:409-12

2. Qudah MA, Bataineh AB. A retrospective study of selected oral and maxillofacial fractures in a group of Jordanian children. Oral Surg Oral Med Oral Pathol Oral Radiol Endod. 2002; 94:310-4

3. Kieser J, Stephenson S, Liston PN, Tong DC, Langley JD. Serious facial fractures in New Zealand from 1979 to 1998. Int J Oral Maxillofacial Surg. 2002; 31:206-9

4. Haug RH, Prather J, Indresano T. An epidemiologic survey of facial fractures and concomitant injuries. J Oral Maxillofac Surg. 1990; 48:926-32

5. Koorey AJ, Marshall SW, Treasure ET, Langley JD. Incidence of facial fractures resulting in hospitalization in New Zealand from 1979 to 1988. Int J Oral Maxillofac Surg. 1992; 21:77-9.

6. Gali R, Devireddy SK, Kumar RK, Kanubaddy SR et al. Faciomaxillary fractures in a semi Urban South Indian Teaching Hospital . A retrospective analysis of 638 cases. Contemporary 
Clinical Dentistry 2015; 6:539 .

7. Garkoti PD, Saklani K, Sharma T, Shashi. A descriptive study of fracture distribution in faciomaxillary trauma in Kumaon region. Journal of Evolution of Medical and Dental Sciences .2015; 4(59):10270-6

8. Sawhney CP, Ahuja R.B. Faciomaxillary fractures in North India: A statistical analysis and review of management . British J Oral Maxillofacial Surg. 1988; 26(5):430-4

9. Adeyemo WL, Ladeinde AL, Ogunlewe MO, James O. Trends and characteristics of oral and maxillofacial injuries in Nigeria: A review of the literature. Head Face Med. 2005;1:7

10. Motamedi MH. An assessment of maxillofacial fractures: A 5-year study of 237 patients. J Oral Maxillofac Surg. 2003; 61:61-4

11. Dutta SRB, Soni S, Prakash R. A study on traumatic faciomaxillary fractures encountered at a tertiary care centre of north-eastern India . BJOHNS 2018; 26(2):79-85

12. Brown RD, Cowpe JG. Patterns of maxillofacial trauma in two different cultures. J R Coll Surg Edinb. 1985; 30:299-302

13. Ansari MH. Maxillofacial fractures in Hamedan province, Iran: a retrospective study (1987-2001) J Cranio maxillofac Surg. 2004; 32:28-34

14. Al Ahmed HE, Jaber MA, Abu Fana SH, Karas M. The pattern of maxillofacial fractures in Sharjah, United Arab Emirates: a review of 230 cases. Oral Surg Oral Med Oral Pathol Oral Radiol Endod. 2004; 98:166-70. doi: 0.1016/j.tripleo.2004.01.020
15. Erol B, Tanrikulu R, Gorgun B. Maxillofacial fractures: Analysis of demographic distribution and treatment in 2901 pateints (25-year expereience) J Craniomaxillofac Surg. 2004; $32: 308-13$

16. Oji C. Jaw fractures in Enugu, Nigeria, 1985-1995. Br J Oral Maxillofac Surg. 1999; 37:106-9. doi: 10.1054/ bjom. 1997.0083

17. Kelly DE, Harrigan WE. A survey of facial fractures: Bellevue Hospital, 1948-1974. J Oral Surg. 1975; 33:146-9

18. Ugboko VI, Odusanya SA, Fagade OO. Maxillofacial fractures in a semi-urban Nigerian teaching hospital. A review of 442 cases. Int J Oral Maxillofac Surg. 1998; 27:286-9

19. Olasoji HO, Tahir A, Bukar A. Jaw fractures in Nigerian children: an analysis of 102 cases. Cent Afr J Med. 2002; 48:109-12

20. Tuli T, Hachl O, Rasse M, Kloss F, Gassner R. Dentoalveolar trauma analysis of 4763 patients with 6237 injuries in 10 years. Mund Kiefer Gesichtschir. 2005; 9(5):324-9

21. Gassner R, Tuli T, Hachl O, Rudisch A, Ulmer H. Craniomaxillofacial trauma: a 10 year review of 9,543 cases with 21,067 injuries. J Craniomaxillofac Surg. 2003; 31:51-61

22. Gassner R, Tuli T, Hachl O, Moreira R, Ulmer $H$. Craniomaxillofacial trauma in children: a review of 3,385 cases with 6,060 injuries in 10 years. J Oral Maxillofac Surg. 2004; 62:399-407. doi: 10.1016/j.joms.2003.05.013. 


\section{Annexure}

\section{Proforma for clinical notes of maxillofacial injuries}

1. Name-

2. Age-

3. Sex-

4. Date of incident-

5. Time of incidence-

6. Place of incidence-

7. Mode of accident-

a. Falling down from vehicle

b. Collision with stationary object

c. Collision with moving vehicle from front

d. Collision with moving vehicle from behind

e. Collision with moving vehicle from side

f. Collision with street animals

g. Others

8. Vehicle in which injured person was traveling
a. Unmotorised two wheelers
b. Motorised two wheelers
c. Four wheelers
d. Not applicable(if injured person was walking)
e. Others

9. In case of collision, type of vehicle with which collision took place
a. Motorised two wheelers
b. Unmotorised two wheelers
c. Four wheelers
d. Others

10. Position of injured person in vehicle
a. Driver of two wheeler
b. Positioned behind driver in two wheeler
c. Front seat of four wheelers
d. Rear seat of four wheelers
e. Positioned elsewhere in a bigger vehicle

12. State of intoxication
a. Of injured person-yes/no
b. Of driver of vehicle if injured person is not the driver-yes/no
c. Driver of colliding vehicle (of known)-yes/no

13. Type of road where incident occurred
a. Kuccha village road
b. Pucca village road
c. Street roads
d. Highway

14. Use of personal safety measures
a. Use of helmets-yes/no/not applicable

b. Use of seat belts-yes/no/not applicable

15. Loss of consciousness-yes/no

16. Vomiting-yes/no

17. Convulsions-yes/no

18. Types of injury

A. Abrasion-present/absent. If present, site-

B. Bruise-present/absent. If present, site-

C. Laceration-present/absent. If present, site-

D. Incised wound-present/absent. If present, site-

E. Cartilage fracture of ear-present/absent

F. Gross nasal bone fracture-present/absent

G. Septal hematoma-present/absent

H. CSF rhinorrhea-present/absent

I. Penetrating injury over neck-present/absent

J. Laryngo-tracheal framework injury-present/absent

K. Epistaxis-present/absent

L. Emphysema-present/absent

19. Radiological injuries
A. Intra cranial hemorrhage
B. Isolated nasal bone fracture
C. Isolated maxillary fracture
D. Isolated Temporal bone fracture
E. Multiple facial bone fracture
F. Dental injury
G. Laryngo-tracheal injury

20. GCS at the time of admission 